\title{
Structural elucidation of metabolites of synthetic cannabinoid UR-144 by Cunninghamella elegans using nuclear magnetic resonance (NMR) spectroscopy
}

Shimpei Watanabe ${ }^{\mathrm{a}}$, Unnikrishnan Kuzhiumparambila ${ }^{\mathrm{a}, \mathrm{b}}$ and Shanlin $\mathrm{Fu}^{\mathrm{a}^{*}}$

${ }^{a}$ Centre for Forensic Science, School of Mathematical and Physical Sciences, University of Technology Sydney (UTS), PO Box 123, Broadway, NSW 2007, Australia

${ }^{b}$ Climate Change Cluster, University of Technology Sydney (UTS), PO Box 123, Broadway, NSW 2007, Australia

Running head: NMR characterisation of UR-144 metabolites

* Corresponding author: Shanlin Fu

Mailing address: Centre for Forensic Science

School of Mathematical and Physical Sciences

University of Technology Sydney (UTS)

PO Box 123, Broadway NSW 2007 Australia

E-mail: Shanlin.fu@uts.edu.au

Phone: +61 295148207

Fax: +61 295142260 


\begin{abstract}
The number of new psychoactive substances keeps on rising despite the controlling efforts by law enforcement. Although metabolism of the newly emerging drugs is continuously studied to keep up with the new additions, the exact structures of the metabolites are often not identified due to the insufficient sample quantities for techniques such as nuclear magnetic resonance (NMR) spectroscopy. The aim of the study was to characterise several metabolites of the synthetic cannabinoid (1-pentyl-1H-indol-3-yl)(2,2,3,3-tetramethylcyclopropyl)methanone (UR-144) by NMR spectroscopy after the incubation with the fungus Cunninghamella elegans. UR-144 was incubated with C. elegans for $72 \mathrm{~h}$ and the resulting metabolites were chromatographically separated. Six fractions were collected and analysed by NMR spectroscopy. UR-144 was also incubated with human liver microsomes (HLM), and the liquid chromatography-high resolution mass spectrometry analysis was performed on the HLM metabolites with the characterised fungal metabolites as reference standards. Ten metabolites were characterised by NMR analysis including dihydroxy metabolites, carboxy and hydroxy metabolites, a hydroxy and ketone metabolite, and a carboxy and ketone metabolite. Of these metabolites, dihydroxy metabolite, carboxy and hydroxy metabolites, and a hydroxy and ketone metabolite were identified in HLM incubation. The results indicate that the fungus is capable of producing human-relevant metabolites including the exact isomers. The capacity of the fungus $C$. elegans to allow for NMR structural characterisation by enabling production of large amounts of metabolites makes it an ideal model to complement metabolism studies.
\end{abstract}

KEY WORDS: Synthetic cannabinoid, Metabolism, Cunninghamella elegans, UR-144, NMR

$\begin{array}{ll}\text { Abbreviations } & \\ \text { C. elegans } & \text { Cunninghamella elegans } \\ \text { ESI } & \text { Electrospray ionisation source } \\ \text { gCOSY } & \text { Gradient correlation spectroscopy } \\ \text { gHMBC } & \text { Gradient heteronuclear multiple bond } \\ & \text { correlation } \\ \text { gHSQC } & \text { Gradient heteronuclear single quantum } \\ & \text { correlation } \\ \text { HLM } & \text { Human liver microsomes } \\ \text { HPLC } & \text { High-performance liquid chromatography } \\ \text { LC-QTOF-MS } & \text { Liquid chromatography-quadrupole time-of- } \\ & \text { flight mass spectrometry } \\ M S & \text { Mass spectrometry } \\ N O E S Y & \text { Nuclear Overhauser effect spectroscopy } \\ N M R & \text { Nuclear magnetic resonance } \\ N P S & \text { New psychoactive substances } \\ \text { TMCP } & \text { Tetramethylcyclopropyl } \\ \text { UR-144 } & \text { (1-pentyl-1H-indol-3-yl)(2,2,3,3- } \\ & \text { tetramethylcyclopropyl)methanone }\end{array}$




\section{Introduction}

The emergence of new psychoactive substances (NPS) has been a worldwide problem for the last decade. In 2016, the rate of the emergence of new drugs has slowed down according to the European Monitoring Centre for Drugs and Drug Addiction [1]. The reporting of 66 new drugs in Europe during the year was the fewest within the last 5 years. Nevertheless, the introduction of more than 1 drug per week still poses a significant challenge especially when more than 620 NPS have to be monitored.

In an effort to keep up with the detection of NPS in drug testing, a number of metabolite identification studies have been conducted [2-6]. This is particularly important for drugs that are extensively metabolised such as synthetic cannabinoids, as the parent drugs may not be present in urine, giving a false negative result [7]. Identification of metabolites can also contribute to the more comprehensive determination of the drug safety, since some metabolites can retain significant pharmacological activities, complicating the adverse effects of the drugs [8].

Several in vivo and in vitro models are commonly used to identify metabolic profiles. Among the in vivo models, controlled self-administration of drugs is a rare strategy due to the health risks and ethical concerns, although it naturally gives the most reliable data [9]. Other in vivo models involve the use of animals such as rats and mice. These models offer the advantage of metabolism occurring within whole animal organisms similar to the human body, although they can be expensive and ethically undesirable, in addition to species differences [6,10]. The most common in vitro model is human liver microsomes (HLM), which are relatively cheap and relevant to human metabolism. However, the lack of some enzymes present in cells can limit the ability to produce relevant in vivo metabolites including phase II metabolites. Also, the typical reaction condition, which contains excessive oxygen, may limit reductive transformation pathways, even when HLM has the appropriate enzyme for the reaction [6]. In contrast, human primary hepatocytes generally provide metabolite profiles that resemble in vivo human profiles more closely, as they are living cells with a closer environment to the human body than enzymes [11].

Although the aforementioned models provide invaluable metabolic data, they have one common limitation: production of small amounts of metabolites. With the recent advances in sensitivity of mass spectrometric techniques, the amounts obtained with these models generally suffice for mass spectrometric analysis. However, mass spectrometric analysis alone is often not adequate for unequivocal structural elucidation including the exact positions of modifications made, leading to the need for analysis by other techniques such as nuclear magnetic resonance (NMR) spectroscopy [11]. Unfortunately, NMR spectroscopy is still a relatively insensitive technique, for which the amount of metabolites obtained from the common models is usually insufficient. Therefore, currently for the unambiguous characterisation of metabolites using these models, it is vital to use reference standards to compare the retention time and mass fragmentation pattern in mass spectrometry.

The fungus Cunninghamella elegans (C. elegans) has been investigated for its capability to mimic human metabolism for decades and is suggested to provide the solution for producing sufficient quantities of metabolites [10]. C. elegans can produce a wide variety of metabolites in a similar manner to humans and incubation of $C$. elegans culture can easily be upscaled, 
allowing large production of metabolites. The advantage of large quantities of metabolites obtained from C. elegans has been proven useful in studies reporting structural characterisation of metabolites using NMR spectroscopy $[12,13]$. We have previously demonstrated, using liquid chromatography - high resolution mass spectrometry, that C. elegans incubation of synthetic cannabinoids, including (1-pentyl-1H-indol-3-yl)(2,2,3,3tetramethylcyclopropyl)methanone (UR-144), can result in similar metabolic profiles to human metabolism $[14,15]$.

In this paper, we report, for the first time using NMR spectroscopy, the structural elucidation of several metabolites of UR-144, which was among the five most commonly seized synthetic cannabinoids in Europe in 2015 [1]. UR-144 was also incubated with HLM and the characterised fungal metabolites were used as reference standards to determine whether these fungal metabolites are produced in HLM incubation.

\section{Materials and methods}

\section{Chemicals and reagents}

UR-144 was synthesised in-house following previously reported methods and characterised by mass spectrometry (MS) and 1D, 2D NMR spectroscopy [16, 17]. LC-MS grade acetonitrile was obtained from Honeywell (Muskegon, MI, USA). Reagent grade dichloromethane and sodium chloride were from Chemsupply (Gilman, SA, Australia). LC-MS grade formic acid and deuterated chloroform $(99.8$ atom $\% \mathrm{D}$, containing $0.03 \%(\mathrm{~V} / \mathrm{V}) \mathrm{TMS})$ was purchased from Sigma-Aldrich (St. Louis, MO, USA). C. elegans ATCC 10028b was from Cryosite Ltd. (South Granville, NSW, Australia). Glycerol and potassium dihydrogen phosphate and dipotassium hydrogen phosphate were obtained from Ajax Chemicals (Auburn, NSW, Australia). Potato dextrose agar, glucose, peptone, and yeast extract were purchased from Oxoid Australia (Adelaide, SA, Australia). HLM 50-Doner pool, NADPH system solution A and NADPH system solution B were obtained from Corning (Corning, NY, USA).

\section{Fungus metabolite characterisation}

\section{Fungus incubation}

Incubation of UR-144 with C. elegans was performed as described in the previous study [14], with the following deviations. A total of $5 \mathrm{~L}$ growth media in multiple conical flasks were used to incubate $50 \mathrm{mg}$ of UR-144. Following liquid-liquid extraction using dichloromethane, the combined extracts were evaporated to dryness and reconstituted in $14 \mathrm{~mL}$ of water:acetonitrile $(55: 45, \mathrm{v} / \mathrm{v})$.

\section{Preparative high-performance liquid chromatography (HPLC)}

HPLC system consisted of an Agilent 1290 LC system with an Agilent Zorbax Eclipse XDBC18 semi-preparative column $(250 \mathrm{~mm} \times 9.4 \mathrm{~mm}, 5 \mu \mathrm{m})$ and an Agilent 1260 Infinity diode array detector. A hundred microlitre of the sample was injected into HPLC to separate some 
UR-144 metabolites, and six fractions were collected, after diode array detection at $254 \mathrm{~nm}$, to obtain purified metabolites. The mobile phase consisted of $0.1 \%$ formic acid in water (A) and $0.1 \%$ formic acid in acetonitrile (B) at a flow rate of $4.0 \mathrm{~mL} / \mathrm{min}$. A run was performed over $18 \mathrm{~min}$ in gradient mode with $45 \% \mathrm{~B}$ from 0 to $10 \mathrm{~min}$, ramped to $95 \% \mathrm{~B}$ at $11 \mathrm{~min}$ and held till $14 \mathrm{~min}$, and ramped back to $45 \% \mathrm{~B}$ at $15 \mathrm{~min}$ and held till $18 \mathrm{~min}$. The column temperature was kept at $30^{\circ} \mathrm{C}$. The procedure was repeated 100 times to obtain sufficient amount of metabolites for NMR analysis. The fractions were placed under a gentle stream of nitrogen gas to remove acetonitrile and then freeze-dried. The samples were redissolved in $\mathrm{CDCl}_{3}$ and analysed by NMR.

\section{NMR spectroscopy}

NMR spectra were acquired at room temperature on an Agilent $500 \mathrm{MHz}$ NMR spectrometer coupled with an Agilent 7510-AS automated NMR sample changer. UR-144 and its metabolites were characterised by ${ }^{1} \mathrm{H} \mathrm{NMR},{ }^{13} \mathrm{C} \mathrm{NMR}$, gradient correlation spectroscopy (gCOSY), gradient heteronuclear single quantum correlation (gHSQC), gradient heteronuclear multiple bond correlation ( $\mathrm{gHMBC}, \mathrm{jnxh}=8 \mathrm{~Hz}$ ), and nuclear Overhauser effect spectroscopy (NOESY) experiments. Acquisition was performed at the frequency of 499.86 and $125.70 \mathrm{MHz}$ for ${ }^{1} \mathrm{H}$ and ${ }^{13} \mathrm{C} \mathrm{NMR}$, respectively. Chemical shifts are reported in $\delta(\mathrm{ppm})$ and were measured relative to $\mathrm{CDCl}_{3}\left({ }^{1} \mathrm{H} \delta=7.26,{ }^{13} \mathrm{C} \delta=77.0\right)$.

\section{Liquid chromatography-quadrupole time-of-flight mass spectrometry (LC-QTOF-MS)}

Each metabolite isolated by preparative HPLC was analysed by LC-QTOF-MS in the same manner as described in the previous study [14], to confirm the identity of the metabolites and compare with the results in the study.

\section{HLM metabolite characterisation}

\section{HLM incubation}

UR-144 solution in acetonitrile:water $(40: 60 \mathrm{v} / \mathrm{v}, 1 \mathrm{mg} / \mathrm{mL}, 25 \mu \mathrm{L})$, phosphate buffer $(0.1 \mathrm{M}$, pH7.4, $855 \mu \mathrm{L})$, NADPH-A $(50 \mu \mathrm{L})$ and NADPH-B $(20 \mu \mathrm{L})$ were mixed in an Eppendorf tube, to which HLM $(50 \mu \mathrm{L})$ was added. The final concentration of acetonitrile was $1 \%$. The mixture was incubated at $37^{\circ} \mathrm{C}$ in a shaking water bath for $3 \mathrm{~h}$. Ice-cold acetonitrile $(1 \mathrm{~mL})$ was added to the mixture for quenching and the Eppendorf tube was centrifuged at $16,060 \times \mathrm{g}$ for $10 \mathrm{~min}$. The supernatant was filtered with a $0.22 \mu \mathrm{m}$ filter. Two microlitre was injected into LC-QTOFMS. A degradation control sample without HLM and a control without UR-144 were also incubated and analysed.

\section{LC-QTOF-MS}

LC-QTOF-MS experiments were performed as described above with the following exceptions. Some analyses were performed on an Agilent 6550A iFunnel Q-TOF with a Dual AJS ESI 
source in Auto MSMS mode, operated with the same parameters as for the fungus analysis except for the following: gas temperature, $290^{\circ} \mathrm{C}$; gas flow, $11 \mathrm{~L} / \mathrm{min}$; sheath gas temperature, $350{ }^{\circ} \mathrm{C}$; sheath gas flow, $11 \mathrm{~L} / \mathrm{min}$; max precursors per cycle, 5 ; static exclusion ranges, 100 300 and 500-1000.

\section{Results}

\section{Fungus metabolite characterisation}

After incubation of UR-144 with C. elegans, the metabolites were separated by HPLC. Based on the abundance and separation of the peaks, six fractions (labelled as fraction 1- fraction 6 below) containing one peak each were isolated and collected for further analysis, apart from fractions 2 and 3 where two peaks partially coeluted and could not be completely separated (Fig. 1). LC-QTOF-MS analysis revealed that fraction 1 gave rise to a single peak which was labelled as U7 in our previous study [14], attributing to metabolite(s) of dihydroxylation. Under the analytical conditions employed for LC-QTOF-MS analysis, the peaks in fractions 2 and 3 eluted as a single peak (U10) corresponding to metabolite(s) with carboxylation and hydroxylation. Fraction 4 was a metabolite with hydroxylation and ketone formation (U14). Fraction 5 corresponded to U15, another metabolite with carboxylation and hydroxylation. Fraction 6 was a metabolite with carboxylation and ketone formation (U17).

\section{NMR analysis}

In total, 10 metabolites were characterised by NMR from six fractions as shown in Fig. 2 (fraction 1 contained M1-M3 (U7); fraction 2, M4 (U10); fraction 3, M5, M6 (U10); fraction 4, M7 (U14); fraction 5, M8, M9 (U15); fraction 6, M10 (U17)). The assigned ${ }^{1} \mathrm{H}$ and ${ }^{13} \mathrm{C}$ NMR data of UR-144 and the metabolites are presented in Tables I - III. The gCOSY and gHMBC correlations, and selected NOE correlations are depicted in Fig. $\mathbf{3}$ and were used to aid assignment of some overlapped ${ }^{1} \mathrm{H}$ and ${ }^{13} \mathrm{C}$ NMR signals in Tables I-III.

\section{HLM metabolite characterisation}

Based on the comparison of retention time and fragmentation pattern in MSMS scan with the fungal metabolites, dihydroxy metabolite (U7), carboxy and hydroxy metabolites (U10 and U15), and hydroxy and ketone metabolite (U14) were identified after HLM incubation. 


\section{Discussion}

\section{Fungus metabolite characterisation}

According to the LC-QTOF-MS analysis in MSMS mode, the following metabolites were identified: dihydroxy metabolite(s) (U7) in fraction 1, carboxylation and hydroxylation metabolites (U10) in fractions 2 and 3 and (U15) in fraction 5, a hydroxylation and ketone formation metabolite (U14) in fraction 4, and a carboxylation and ketone formation metabolite (U17) in fraction 6. To determine the exact positions of the modifications in these metabolites, 1D and 2D NMR data was obtained including ${ }^{1} \mathrm{H},{ }^{13} \mathrm{C}, \mathrm{gCOSY}, \mathrm{gHSQC}, \mathrm{gHMBC}$, and NOESY spectra.

\section{NMR analysis}

UR-144

First, the parent drug UR-144 was analysed (Table I). NMR data of UR-144 have been previously reported with some discrepancies in signal assignments among the studies [18-20]. Our results agree well with the assignments given by Langer et al. [18]. After the analysis of UR-144, each fraction containing metabolites was analysed. Since all the metabolites were determined to have the intact indole moiety based on LC-QTOF-MS, the signals related to the indole moiety are generally not discussed below.

\section{Dihydroxy metabolites M1-M3 (U7)}

LC-QTOF-MS analysis of fraction 1 (U7) tentatively assigned the single peak (RT $=6.9$ min, Fig. 1) as dihydroxy metabolite(s), with one hydroxy group located at the tetramethylcyclopropyl (TMCP) ring and the other one at the pentyl side chain, as reported in the previous study [14]. NMR study has shown that this fraction in fact contained three isomers designated as M1, M2, and M3, respectively (Table I). The NMR data shows that hydroxylation at the TMCP ring occurred at one of the four methyl groups instead of the C-1"' adjacent to the carbonyl group. This assignment was based on two observations. Firstly, the C-1"' was found to retain its hydrogen resonating around $\delta 2.12 \mathrm{ppm}$. Secondly, the appearance of the signals at $\delta 3.77 \mathrm{ppm}$ as a doublet of doublets was attributable to the diastereotopic methylene protons following hydroxylation at one of the four methyl groups (2"'-Me, or 3"'-Me); the strong shift of the signal from around $\delta 1.30 \mathrm{ppm}$ to the low magnetic field by approximately $\delta 2.45 \mathrm{ppm}$ is in line with the electron withdrawing effect of the added hydroxyl group. NOESY cross peaks at $\delta 2.12-2.13(\mathrm{H}-1 " ') / \delta 3.77\left(\mathrm{HO}-\mathrm{CH}_{2}-(E)-2^{\prime \prime \prime}\right)$ and $\delta 2.12-2.13\left(\mathrm{H}-1^{\prime \prime \prime}\right) / \delta 1.36-1.39\left(\mathrm{CH}_{3}-\right.$ $(E)-3 " ')$ suggests that the metabolites are E-isomers in that the hydroxy group is in an orientation trans to the adjacent carbonyl group (Fig. 3, M1-M3). Based on the results, the hydroxy group can be either at $(E)-2$ "'-Me or $(E)-3$ "'-Me. For simplicity, the hydroxy group is shown at $(E)-2 " '-M e$ only, as the ring structure is otherwise symmetrical. Similarly, hydroxylation and carboxylation for the subsequent metabolites will also be shown at 2"'-Me only rather than 2"'-Me and 3"'-Me.

In contrast to the TMCP ring, hydroxylation at the pentyl side chain occurred at two different positions. COSY and HMBC correlations showed two spin systems for the side chain as shown 
in Fig. 3 (M1-M3). One of them is a 5-hydroxypentyl metabolite (M1), which is supported by C-5" having two hydrogens instead of three as indicated by an HSQC cross peak (data not shown). The other one is a 4-hydroxypentyl metabolite, which is supported by H-5" being a doublet. While 5-hydroxypentyl chain is achiral, 4-hydroxypentyl chain has a chiral centre. Therefore, 4-hydroxypentyl chain can exist as enantiomers with C-4" having $(R)$ or $(S)$ configuration. In addition, the hydroxylated TMCP ring determined above also has a chiral carbon at $(E)-2$ "' when $(E)-2$ "'-Me is hydroxylated, i.e. $(S)$-isomer, whereas the metabolite with a hydroxy group at $(E)-3$ "'-Me has the $(R)$ configuration. Hence, there can be four stereoisomers for the dihydroxy metabolites containing 4-hydroxypentyl chain and a hydroxy group at $(E)$ 2"'-Me or (E)-3"'-Me. Of the four stereoisomers, (4"R, 3"'R) and (4"S, 2"'S) are enantiomers as well as (4"R, 2"'S) and (4"S, 3"'R). Since enantiomers are not differentiated by NMR spectra, only the two diastereomers should appear in the spectra as observed in the study (M2 and M3). All three metabolites are similar in structure and account for the coelution in LC separation.

\section{Hydroxy and ketone metabolite M7 (U14)}

Fraction 4 (M7) showed proton and carbon signals almost identical to M2 and M3 for the TMCP ring indicating hydroxylation at (E)-2"'-Me (Table III). The distinct carbon signal at $\delta$ 207.5 ppm suggested the presence of a ketone group, which is consistent with the MS findings. The proton signals for the pentyl side chain showed one singlet, two triplets and one quintet. A singlet can occur only when C-2" or C-4" has a ketone group. If C-2" had a ketone group, H-1" would become a singlet. However, there was a triplet at $\delta 4.21 \mathrm{ppm}$ corresponding to $\mathrm{H}-1$ " as seen for UR-144 and the dihydroxy metabolites, which concludes the position of the ketone group to be at C-4" of the pentyl side chain. The cross peaks in COSY and HMBC also supported the location of the ketone group to be C-4" (Fig. 3). The metabolite M7 is an oxidised metabolite of M2 and/or M3 (Fig. 2).

\section{Carboxy and hydroxy metabolites M4-M6 (U10) and M8-M9 (U15)}

Since fractions 2 and 3 were collected from the coeluting peaks in preparative HPLC, these fractions were expected to contain a few metabolites originating from the two coeluting peaks. Indeed, they contained signals from both peaks, but the relative abundance of the signals made it clear enough for assigning which signals were from fraction 2 or fraction 3. As expected from MS analysis, fraction 2 (M4) showed a carbon signal at $\delta 173.6 \mathrm{ppm}$ for carboxylic acid in the TMCP ring (Table II). Based on the structure of the TMCP ring, the possible position for carboxylation is at one of the four methyl groups, i.e., 2"'-Me or 3"'-Me. NOESY cross peaks at $\delta 2.38\left(\mathrm{H}-1^{\prime \prime \prime}\right) / \delta 1.56\left(\mathrm{CH}_{3}-(E)-2^{\prime \prime \prime}\right)$ and $\delta 2.38\left(\mathrm{H}-1^{\prime \prime \prime}\right) / \delta 1.50\left(\mathrm{CH}_{3}-(E)-3^{\prime \prime \prime}\right)$ indicated that the carboxy group is cis to the carbonyl group (Fig. 3). The hydroxy group at the pentyl side chain was assigned at H-5" based on the COSY and HMBC data (Fig. 3). The proton and carbon data for the side chain was analogous to those of M1, as M1 is also hydroxylated at $\mathrm{H}$ $5 "$.

Having coeluted with a peak in fraction 2, a peak in fraction 3 is expected to have structurally similar compounds. In fact, fraction 1 displayed a similar case where 5-hydroxypentyl metabolite with another hydroxy group at the TMCP ring coeluted with two diastereomers of 4-hydroxypentyl metabolites having another hydroxy group at the TMCP ring. As expected, 
fraction 3 showed the presence of 4-hydroxypentyl metabolite with a carboxy group at the TMCP ring (M5 and M6); the carboxy group was found cis to the carbonyl group in the TMCP ring as indicated by NOSEY cross peaks at $\delta 2.38\left(\mathrm{H}-1{ }^{\prime \prime \prime}\right) / \delta 1.56\left(\mathrm{CH}_{3}-(E)-2^{\prime \prime \prime}\right)$ and $\delta 2.38(\mathrm{H}-$ $\left.1^{\prime \prime \prime}\right) / \delta 1.50\left(\mathrm{CH}_{3}-(E)-3^{\prime \prime \prime}\right)$, and the hydroxy group was assigned to $\mathrm{H}-4$ " by COSY and HMBC cross peaks (Fig. 3), and the proton and carbon data was similar to that of M2 and M3. As was the case with M2 and M3, M5 and M6 can have two diastereomers due to the presence of two stereo centres, and hence $\mathrm{H}-2$ ' and some carbon atoms showed two signals corresponding to the presence of diastereomers.

Fraction 5 (M8 and M9) showed the proton and carbon signals for the TMCP ring, almost identical to those of M4-M6, indicating the carboxy group is cis to the carbonyl group (Table II, Fig. 3). Now that M4, and M5 and M6 were characterised as 5-hydroxypentyl and 4hydroxypentyl metabolites, respectively, the position of the hydroxy group in the pentyl side chain for this fraction can only be at H-1", H-2" or H-3". Regardless of the exact position, the metabolite would have two stereo centres, leading to two diastereomers as described above. Indeed, H-2' showed two signals resulting from the diastereomers. The cross peaks in COSY and $\mathrm{HMBC}$ revealed the position of hydroxylation to be at H-3", which is supported by the fact that the proton peak at $\delta 4.42$ (H-1") is a triplet, which would be a doublet for a 2-hydroxypentyl metabolite, and the peak has two protons according to the HSQC data and hence cannot be hydroxylated at H-1".

Carboxy and ketone metabolite M10 (U17)

Fraction 6 (M10) showed similar proton, carbon and 2D NMR data to M4, M5, M6, M8, and M9 for the TMCP ring, indicating carboxylation at a methyl group cis to the carbonyl group (Table III, Fig. 3). For the pentyl side chain, the ketone carbon signal at $\delta 207.2$ ppm together with proton and carbon data similar to M7 allowed the position of ketone formation to be C4". This metabolite M10 is considered to be derived from further oxidation of M5 and/or M6 (Fig. 2). It is interesting to note that the chemical shift of carbon signals at C-4', C-5' and C-6' in the indole moiety were in the order of C-4' $<\mathrm{C}-5^{\prime}<\mathrm{C}-6^{\prime}$ for M10 and all the other metabolites with a carboxy group at the TMCP ring, whereas it was in the order of C-5' $<$ C-4' $<$ C-6' for UR-144 and the other metabolites.

\section{HLM metabolite characterisation}

Using the isolated and characterised fungal metabolites as reference standards, dihydroxy metabolite (U7/M1-M3), carboxy and hydroxy metabolites (U10/M4-M6 and U15/M8-M9), and hydroxy and ketone metabolite (U14/M7) were able to be identified in the HLM sample. Under the chromatographic conditions employed, 5-hydroxypentyl and 4-hydroxypentyl metabolites (M1-M3 and M4-M6) coeluted, and hence, there can be both or either of the metabolites. The presence of these metabolites was previously reported in human metabolism as well as the fungal metabolism: dihydroxy metabolites and hydroxy and ketone metabolites in human urine; dihydroxy metabolites and carboxy and hydroxy metabolites in HLM; all the metabolites in the fungus [14, 21-24]. However, it was not possible to determine if the human metabolites were the same as the exact isomers found in the fungus without analysing them by the same LC-MS system. Given that the exact isomers of the fungal metabolites were identified 
after HLM incubation, the fungal metabolites can be a powerful tool to characterise human metabolites.

\section{Advantages and disadvantages of the fungus metabolism}

The main advantage of the fungus $C$. elegans is that experiments can easily be upscaled, which would allow NMR analysis of metabolites. As mentioned before, the NMR analysis of metabolites is imperative for comprehensive characterisation of most metabolites without using reference standards. This proves even more important for the metabolites which are not commercially available. As of now, there are only six metabolites available for UR-144 from Cayman Chemical: $N$-pentanoic acid metabolite, $N$-(5-hydroxypentyl) metabolite, $( \pm)-N-(4-$ hydroxypentyl) metabolite, $N$-(2-hydroxypentyl) metabolite, $N$-(5-hydroxypentyl) $\beta$-Dglucuronide, and UR-144 degradant $N$-pentanoic acid metabolite [25]. Also, the present study has demonstrated that NMR analysis can detect two or more isomers, which cannot be differentiated by MS, in a reasonably symmetrical peak in a chromatogram. Therefore, to fully characterise other metabolites including M1-M10, analysis by NMR spectroscopy is essential, and this could be provided by a large-scale fungus experiment. The limitations of the largescale fungus experiment include the difficulty in separating closely structured isomers using preparative HPLC, and the amounts of metabolites obtained are still generally less than those obtained by synthesis, making the NMR interpretation more challenging. However, chromatographic separations may be significantly improved by the use of different HPLC columns such as chiral columns, and a larger column will make it easier to obtain larger amounts of metabolites. Unlike organic synthesis, several metabolites can be obtained in one incubation, in which the fungus "synthesises" the metabolites for us, including the metabolites which are difficult to synthesise chemically [10]. Hence, the fungus $C$. elegans can be an invaluable model for studying drug metabolism.

\section{Conclusion}

The synthetic cannabinoid UR-144 was incubated with the fungus C. elegans in a large scale, and several metabolites were isolated by preparative HPLC. Ten metabolites were characterised by NMR analysis, including dihydroxy metabolites, carboxy and hydroxy metabolites, a hydroxy and ketone metabolite, and a carboxy and ketone metabolite. UR-144 was also incubated with HLM, and the characterised fungal metabolites were used as reference standards to determine if these metabolites were generated by HLM. LC-QTOF-MS analysis identified dihydroxy metabolite (U7), carboxy and hydroxy metabolites (U10 and U15), and hydroxy and ketone metabolite (U14) after HLM incubation. These findings demonstrate the ability of the fungus to produce human-relevant metabolites of the synthetic cannabinoid in sufficient abundance for NMR analysis, indicating the potential for the fungus model as a tool to complement metabolism studies.

The study highlights the limitations of LC-MS/MS in identifying metabolites of NPS, an analytical technique widely utilised in carrying out metabolism study. It also illustrates the importance of using other complimentary techniques such as NMR in unambiguous elucidation of metabolite structures. 


\section{Acknowledgments}

The authors would like to thank Jane Cameron and Zofia Winiarski for the support with fungus culturing and Mahmoud El Safadi for the support with organic synthesis.

\section{Conflicts of interest}

The authors declare that they have no conflicts of interest.

\section{References}

1. European Monitoring Centre for Drugs and Drug Addiction. European drug report 2017: Trends and developments. Luxembourg: Publications Office of the European Union 2017.

2. Carlier J, Diao X, Sempio C, Huestis MA. Identification of new synthetic cannabinoid adb-chminaca (mab-chminaca) metabolites in human hepatocytes. AAPS J. 2017:1-10. doi:10.1208/s12248-016-0037-5.

3. Grafinger KE, Hädener M, König S, Weinmann W. Study of the in vitro and in vivo metabolism of the tryptamine 5-meo-mipt using human liver microsomes and real case samples. Drug Test Anal.in press. doi:10.1002/dta.2245.

4. Meyer MR. New psychoactive substances: An overview on recent publications on their toxicodynamics and toxicokinetics. Arch Toxicol. 2016;90(10):2421-44. doi:10.1007/s00204016-1812-x.

5. Watanabe S, Vikingsson S, Roman M, Green H, Kronstrand R, Wohlfarth A. In vitro and in vivo metabolite identification studies for the new synthetic opioids acetylfentanyl, acrylfentanyl, furanylfentanyl, and 4-fluoro-isobutyrylfentanyl. AAPS J. 2017;19(4):1102-22. doi:10.1208/s12248-017-0070-z.

6. Vikingsson S, Wohlfarth A, Andersson M, Gréen H, Roman M, Josefsson M, et al. Identifying metabolites of meclonazepam by high-resolution mass spectrometry using human liver microsomes, hepatocytes, a mouse model, and authentic urine samples. AAPS J. 2017;19(3):736-42. doi:10.1208/s12248-016-0040-x.

7. Diao X, Scheidweiler KB, Wohlfarth A, Pang S, Kronstrand R, Huestis MA. In vitro and in vivo human metabolism of synthetic cannabinoids fdu-pb-22 and fub-pb-22. AAPS J. 2016;18(2):455-64. doi:10.1208/s12248-016-9867-4.

8. Chimalakonda KC, Seely KA, Bratton SM, Brents LK, Moran CL, Endres GW, et al. Cytochrome p450-mediated oxidative metabolism of abused synthetic cannabinoids found in k2/spice: Identification of novel cannabinoid receptor ligands. Drug Metab Dispos. 2012;40(11):2174-84. doi:10.1124/dmd.112.047530.

9. Hutter M, Moosmann B, Kneisel S, Auwarter V. Characteristics of the designer drug and synthetic cannabinoid receptor agonist am-2201 regarding its chemistry and metabolism. J Mass Spectrom. 2013;48(7):885-94. doi:10.1002/jms.3229.

10. Asha S, Vidyavathi M. Cunninghamella--a microbial model for drug metabolism studies--a review. Biotechnol Adv. 2009;27(1):16-29. doi:10.1016/j.biotechadv.2008.07.005.

11. Diao X, Huestis MA. Approaches, challenges, and advances in metabolism of new synthetic cannabinoids and identification of optimal urinary marker metabolites. Clin Pharmacol Ther. 2017;101(2):239-53. doi:10.1002/cpt.534. 
12. Guddat S, Fußhöller G, Beuck S, Thomas A, Geyer H, Rydevik A, et al. Synthesis, characterization, and detection of new oxandrolone metabolites as long-term markers in sports drug testing. Anal Bioanal Chem. 2013;405(25):8285-94. doi:10.1007/s00216-013-7218-1.

13. Tian J-L, Chen Y, Wang Y-X, Huang X-X, Sun X, Liu K-C, et al. Microbial transformation of methyl cyperenoate by cunninghamella elegans as 3.2028 and the antithrombotic activities of its metabolites. RSC Adv. 2016;6(113):112712-20. doi:10.1039/c6ra24332k.

14. Watanabe S, Kuzhiumparambil U, Nguyen MA, Cameron J, Fu S. Metabolic profile of synthetic cannabinoids $5 \mathrm{f}-\mathrm{pb}-22$, pb-22, xlr-11 and ur-144 by cunninghamella elegans. AAPS J. 2017;19(4):1148-62. doi:10.1208/s12248-017-0078-4.

15. Watanabe S, Kuzhiumparambil U, Winiarski Z, Fu S. Biotransformation of synthetic cannabinoids jwh-018, jwh-073 and am2201 by cunninghamella elegans. Forensic Sci Int. 2016;261:33-42. doi:http://dx.doi.org/10.1016/j.forsciint.2015.12.023.

16. Nunomoto S, Kawakami Y, Yamashita Y, Takeuchi H, Eguchi S. Regioselectivity control in alkylation reactions of indolyl ambident anion. J Chem Soc, Perkin Trans 1. 1990(1):111-4. doi:10.1039/P19900000111.

17. Okauchi T, Itonaga M, Minami T, Owa T, Kitoh K, Yoshino H. A general method for acylation of indoles at the 3-position with acyl chlorides in the presence of dialkylaluminum chloride. Org Lett. 2000;2(10):1485-7. doi:10.1021/o1005841p.

18. Langer N, Lindigkeit R, Schiebel H-M, Ernst L, Beuerle T. Identification and quantification of synthetic cannabinoids in 'spice-like' herbal mixtures: A snapshot of the german situation in the autumn of 2012. Drug Test Anal. 2014;6(1-2):59-71. doi:10.1002/dta.1499.

19. Kavanagh P, Grigoryev A, Savchuk S, Mikhura I, Formanovsky A. Ur-144 in products sold via the internet: Identification of related compounds and characterization of pyrolysis products. Drug Test Anal. 2013;5(8):683-92. doi:10.1002/dta.1456.

20. Shevyrin V, Melkozerov V, Nevero A, Eltsov O, Morzherin Y, Shafran Y. Identification and analytical properties of new synthetic cannabimimetics bearing 2,2,3,3tetramethylcyclopropanecarbonyl moiety. Forensic Sci Int. 2013;226(1):62-73. doi:http://dx.doi.org/10.1016/j.forsciint.2012.12.009.

21. Sobolevsky T, Prasolov I, Rodchenkov G. Detection of urinary metabolites of am-2201 and ur-144, two novel synthetic cannabinoids. Drug Test Anal. 2012;4(10):745-53. doi:10.1002/dta.1418.

22. Nielsen LM, Holm NB, Olsen L, Linnet K. Cytochrome p450-mediated metabolism of the synthetic cannabinoids ur-144 and xlr-11. Drug Test Anal. 2016;8(8):792-800. doi:10.1002/dta.1860.

23. Grigoryev A, Kavanagh P, Melnik A, Savchuk S, Simonov A. Gas and liquid chromatography-mass spectrometry detection of the urinary metabolites of ur-144 and its major pyrolysis product. J Anal Toxicol. 2013;37(5):265-76. doi:10.1093/jat/bkt028.

24. Adamowicz P, Zuba D, Sekula K. Analysis of ur-144 and its pyrolysis product in blood and their metabolites in urine. Forensic Sci Int. 2013;233(1-3):320-7. doi:10.1016/j.forsciint.2013.10.005.

25. Cayman Chemical. 2017. https://www.caymanchem.com/Search?q=ur-144. Accessed 27 July 2017. 


\section{Figures}

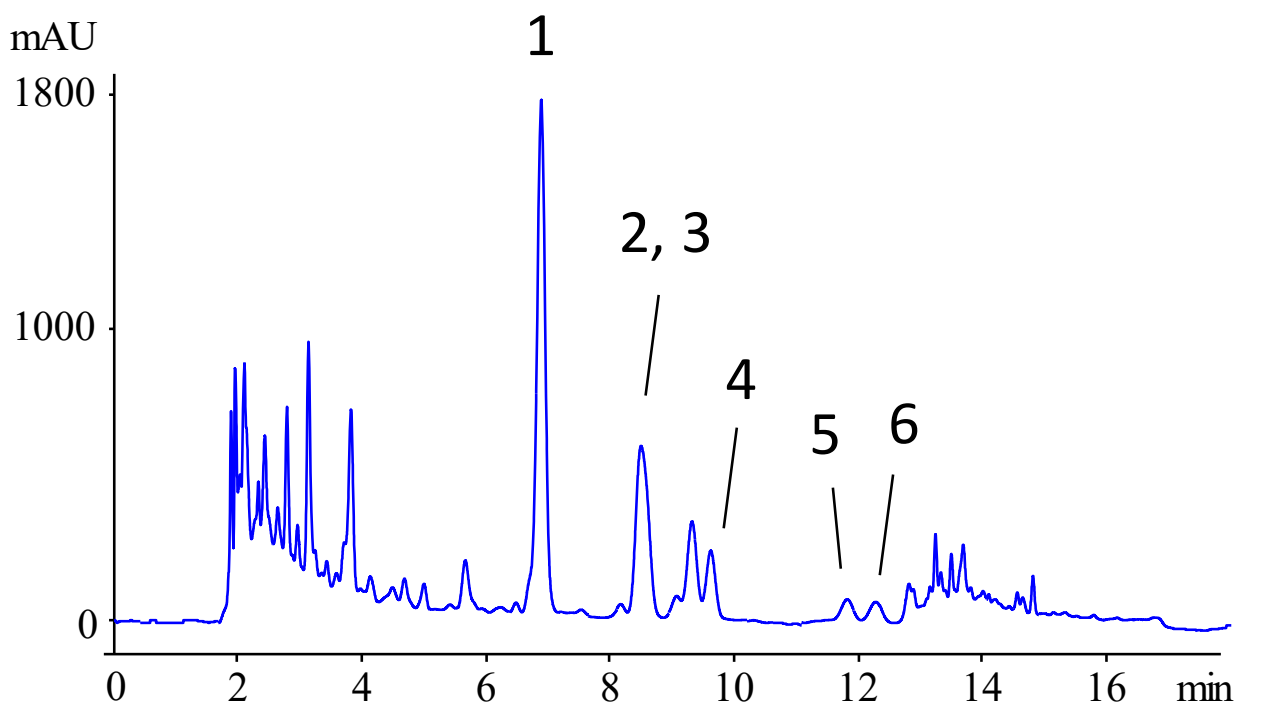

\begin{tabular}{|l|l|l|}
\hline Fraction & NMR & LC-MS/MS (Ref [14]) \\
\hline 1 & M1, M2, M3 & U7 \\
\hline 2 & M4 & U10 \\
\hline 3 & M5, M6 & U10 \\
\hline 4 & M7 & U14 \\
\hline 5 & M8, M9 & U15 \\
\hline 6 & M10 & U17 \\
\hline
\end{tabular}

Fig. 1. Preparative HPLC chromatogram showing separation of fractions 1-6. Fractions 2 and 3 partially coeluted. Summarised here are also the corresponding metabolites identified by NMR and LC-MS/MS in each fraction. 


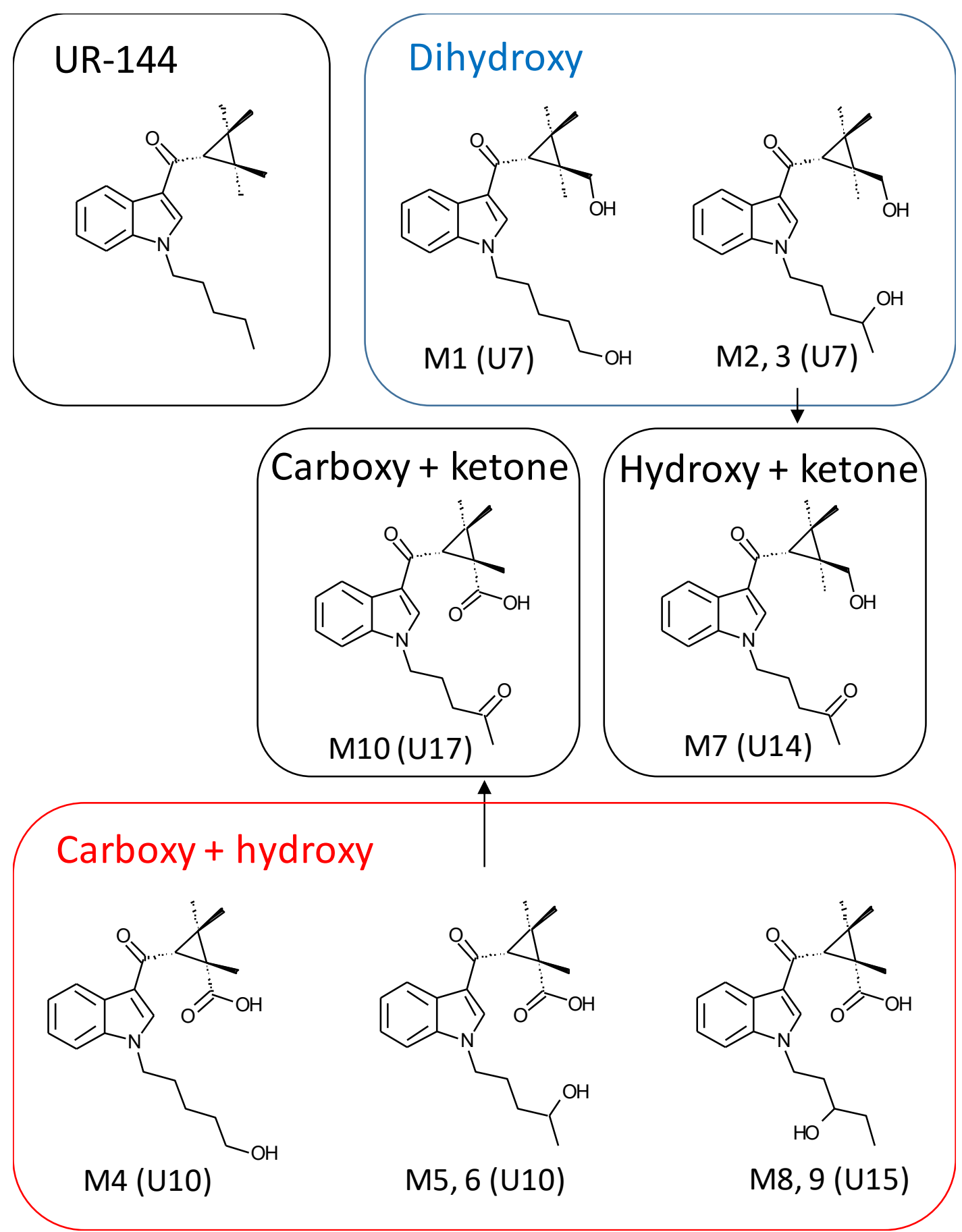

Fig. 2. The structures of UR-144 and the metabolites analysed in this study. Brackets indicate the metabolite ID in our previous paper [14]. Arrows indicate partial metabolic pathways. M2 and M3, M5 and M6, and M8 and M9 are diastereomers, respectively, and share the same structures apart from the spatial configurations at the stereo centres 

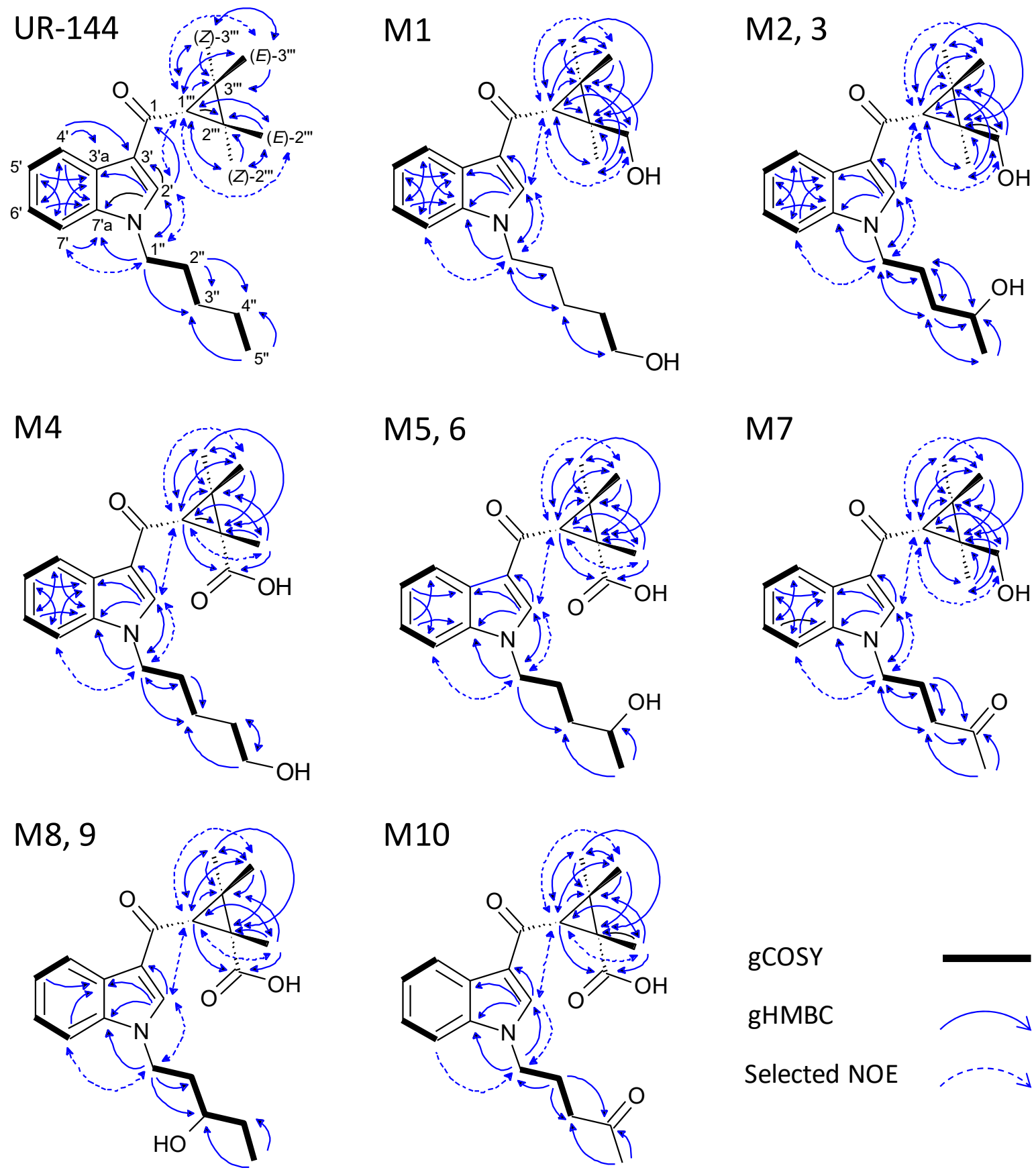

Selected NOE

Fig. 3. Gradient correlation spectroscopy (gCOSY), gradient heteronuclear multiple bond correlation (gHMBC), and selected nuclear Overhauser effect (NOE) correlations of UR-144 and the metabolites analysed in the study 


\section{Tables}

Table I. ${ }^{1} \mathrm{H}$ and ${ }^{13} \mathrm{C}$ NMR data for UR-144 and fraction 1 containing dihydroxy metabolites (M1-M3) in $\mathrm{CDCl}_{3}$

\begin{tabular}{|c|c|c|c|c|c|c|}
\hline \multirow{3}{*}{$\begin{array}{l}\text { Position } \\
\text { No. }\end{array}$} & \multicolumn{2}{|c|}{ UR-144 } & \multicolumn{4}{|c|}{ Fraction 1} \\
\hline & & & M1 & M2/M3 & M1 & M2/M3 \\
\hline & ${ }^{13} \mathrm{C}$ & ${ }^{1} \mathrm{H}$ & \multicolumn{2}{|l|}{${ }^{13} \mathrm{C}$} & \multicolumn{2}{|l|}{${ }^{1} \mathrm{H}$} \\
\hline 1 & 194.6 & & \multicolumn{2}{|l|}{193.4} & & \\
\hline $2^{\prime}$ & 133.4 & $7.66(s)$ & 134.0 & $\begin{array}{l}134.1 \\
134.4\end{array}$ & $7.83(\mathrm{~s})$ & $\begin{array}{l}7.87(\mathrm{~s}), 7.88 \\
(\mathrm{~s})\end{array}$ \\
\hline $3^{\prime}$ & 119.6 & & \multicolumn{2}{|c|}{$119.1,119.2$} & & \\
\hline 3'a & 126.4 & & \multicolumn{2}{|c|}{$126.38,126.42$} & & \\
\hline $4^{\prime}$ & 122.7 & 8.39-8.41 (m) & \multicolumn{2}{|l|}{122.7} & \multicolumn{2}{|l|}{$8.38-8.40(\mathrm{~m})$} \\
\hline $5^{\prime}$ & 122.0 & $\begin{array}{l}7.23-7.29(\mathrm{~m}) \\
\text { overlapped }\end{array}$ & \multicolumn{2}{|c|}{$122.26,122.28$} & \multicolumn{2}{|c|}{ 7.24-7.30 (m), overlapped } \\
\hline $6^{\prime}$ & 122.8 & $\begin{array}{l}7.23-7.29(\mathrm{~m}) \\
\text { overlapped }\end{array}$ & \multicolumn{2}{|c|}{$123.0,123.1$} & \multicolumn{2}{|c|}{ 7.24-7.30 (m), overlapped } \\
\hline $7^{\prime}$ & 109.6 & $7.33-7.35(\mathrm{~m})$ & \multicolumn{2}{|c|}{$109.60,109.63$} & \multicolumn{2}{|l|}{$7.33-7.37(\mathrm{~m})$} \\
\hline 7 'a & 136.6 & & \multicolumn{2}{|l|}{136.6} & & \\
\hline $1 "$ & 47.0 & $\begin{array}{l}4.14(\mathrm{t}, \mathrm{J}=7.3 \\
\mathrm{Hz})\end{array}$ & 46.9 & 46.8 & $\begin{array}{l}4.20(\mathrm{t}, \mathrm{J}= \\
7.0 \mathrm{~Hz})\end{array}$ & $\begin{array}{l}4.20(\mathrm{t}, \mathrm{J}= \\
7.0 \mathrm{~Hz})\end{array}$ \\
\hline $2^{\prime \prime}$ & 29.6 & $\begin{array}{l}1.89 \text { (quintet, } \mathrm{J}= \\
7.5 \mathrm{~Hz}) \text { ) }\end{array}$ & 29.5 & $\begin{array}{l}26.1 \\
26.2\end{array}$ & $\begin{array}{l}1.93^{\mathrm{a}}, \\
\text { overlapped }\end{array}$ & $\begin{array}{l}1.90-2.10 \\
(\mathrm{~m})\end{array}$ \\
\hline $3 "$ & 29.0 & $\begin{array}{l}1.36^{\mathrm{a}} \\
\text { overlapped }\end{array}$ & 23.2 & $\begin{array}{l}35.9 \\
36.0\end{array}$ & $\begin{array}{l}1.44^{\mathrm{a}}, \\
\text { overlapped }\end{array}$ & $\begin{array}{l}1.49 \text { (quintet, } \\
\mathrm{J}=7.3 \mathrm{~Hz} \text { ) }\end{array}$ \\
\hline $4 "$ & 22.2 & $\begin{array}{l}1.36^{\mathrm{a}}, \\
\text { overlapped }\end{array}$ & 31.9 & $\begin{array}{l}67.6 \\
67.7\end{array}$ & $\begin{array}{l}1.61^{\mathrm{a}} \\
\text { overlapped }\end{array}$ & $\begin{array}{l}3.84(\mathrm{~m}), \\
\text { overlapped }\end{array}$ \\
\hline $5 "$ & 13.9 & $\begin{array}{l}0.91(t, J=7.0 \\
\mathrm{Hz})\end{array}$ & 62.5 & $\begin{array}{l}23.8 \\
23.9\end{array}$ & $\begin{array}{l}3.65(\mathrm{td}, \mathrm{J}= \\
6.3,4.3 \mathrm{~Hz})\end{array}$ & $\begin{array}{l}1.20(\mathrm{~d}, 6.0 \\
\mathrm{Hz})\end{array}$ \\
\hline $1 " '$ & 41.6 & $1.94(\mathrm{~s})$ & \multicolumn{2}{|c|}{$38.76,38.77$} & \multicolumn{2}{|c|}{$2.120(\mathrm{~s}), 2.124(\mathrm{~s}), 2.13(\mathrm{~s})$} \\
\hline $2 " '$ & 31.5 & & \multicolumn{2}{|c|}{$36.0,36.1$} & & \\
\hline 3"' & 31.5 & & \multicolumn{2}{|c|}{$30.0,30.1,30.2$} & & \\
\hline$(E)-2$ "'-Me & 24.0 & $1.31(\mathrm{~s})$ & \multicolumn{2}{|c|}{$69.3,69.4,69.5$} & $\begin{array}{l}3.766(\mathrm{dd}, \mathrm{J} \\
=36.3,11.3 \\
\mathrm{~Hz})\end{array}$ & $\begin{array}{l}3.765(\mathrm{dd}, \mathrm{J} \\
=19.5,11.5 \\
\mathrm{~Hz}), 3.769 \\
(\mathrm{dd}, \mathrm{J}=28.8 \\
11.3 \mathrm{~Hz})\end{array}$ \\
\hline (Z)-2"'-Me & 17.0 & $1.35(\mathrm{~s})$ & \multicolumn{2}{|c|}{$12.4,12.5$} & \multicolumn{2}{|c|}{$1.40(\mathrm{~s}), 1.42(\mathrm{~s}), 1.43(\mathrm{~s})$} \\
\hline (E)-3"'-Me & 24.0 & $1.31(\mathrm{~s})$ & \multicolumn{2}{|c|}{$\begin{array}{l}17.52,17.55 \\
17.57, \text { or } 23.5^{b}\end{array}$} & \multicolumn{2}{|c|}{$\begin{array}{l}1.36(\mathrm{~s}), 1.37(\mathrm{~s}), 1.378(\mathrm{~s}), \\
1.380(\mathrm{~s}), 1.384(\mathrm{~s}), 1.39(\mathrm{~s}), \\
\text { overlapped }\end{array}$} \\
\hline (Z)-3"'-Me & 17.0 & $1.35(\mathrm{~s})$ & \multicolumn{2}{|c|}{$\begin{array}{l}17.52,17.55 \\
17.57, \text { or } 23.5^{b}\end{array}$} & \multicolumn{2}{|c|}{$\begin{array}{l}1.36(\mathrm{~s}), 1.37(\mathrm{~s}), 1.378(\mathrm{~s}), \\
1.380(\mathrm{~s}), 1.384(\mathrm{~s}), 1.39(\mathrm{~s}), \\
\text { overlapped }\end{array}$} \\
\hline
\end{tabular}

${ }^{a}$ The chemical shifts were taken from HSQC data

${ }^{\mathrm{b}}$ Based on the data, it could not be determined which chemical shifts correspond to (E)- and (Z)-methyl carbons 
Table II. ${ }^{1} \mathrm{H}$ and ${ }^{13} \mathrm{C}$ NMR data for carboxy and hydroxy metabolites (M4-6 and M8-9) in $\mathrm{CDCl}_{3}$

\begin{tabular}{|c|c|c|c|c|c|c|}
\hline \multirow{2}{*}{$\begin{array}{l}\text { Position } \\
\text { No. }\end{array}$} & \multicolumn{2}{|l|}{ M4 } & \multicolumn{2}{|l|}{$\mathrm{M} 5 / 6$} & \multicolumn{2}{|l|}{$\mathrm{M} 8 / 9$} \\
\hline & ${ }^{13} \mathrm{C}$ & ${ }^{1} \mathrm{H}$ & ${ }^{13} \mathrm{C}$ & ${ }^{1} \mathrm{H}$ & ${ }^{13} \mathrm{C}$ & ${ }^{1} \mathrm{H}$ \\
\hline 1 & 196.4 & & 196.4 & & ND & \\
\hline $2^{\prime}$ & 136.2 & $7.80(\mathrm{~s})$ & 136.3 & $\begin{array}{l}7.82(\mathrm{~s}), 7.83 \\
(\mathrm{~s})\end{array}$ & 136.8 & $\begin{array}{l}7.85(\mathrm{~s}), 7.88 \\
(\mathrm{~s})\end{array}$ \\
\hline $3^{\prime}$ & 117.2 & & $117.1^{\mathrm{b}}$ & & $117.2^{\mathrm{b}}$ & \\
\hline $3 ' a$ & 126.1 & & 126.1 & & $126.1^{\mathrm{b}}$ & \\
\hline $4^{\prime}$ & 122.7 & $8.32-8.34(\mathrm{~m})$ & 122.7 & $8.32-8.34(\mathrm{~m})$ & 122.7 & $8.32-8.34(\mathrm{~m})$ \\
\hline $5^{\prime}$ & 123.7 & $\begin{array}{l}7.35-7.38 \\
(\mathrm{~m}), \\
\text { overlapped }\end{array}$ & 123.7 & $\begin{array}{l}7.35-7.38 \\
(\mathrm{~m}), \\
\text { overlapped }\end{array}$ & 123.7 & $\begin{array}{l}7.35-7.38 \\
(\mathrm{~m}), \\
\text { overlapped }\end{array}$ \\
\hline $6^{\prime}$ & 124.1 & $\begin{array}{l}7.35-7.38 \\
(\mathrm{~m}), \\
\text { overlapped }\end{array}$ & 124.1 & $\begin{array}{l}7.35-7.38 \\
(\mathrm{~m}), \\
\text { overlapped }\end{array}$ & 124.1 & $\begin{array}{l}7.35-7.38 \\
(\mathrm{~m}), \\
\text { overlapped }\end{array}$ \\
\hline $7^{\prime}$ & 110.2 & $7.40-7.44(\mathrm{~m})$ & 110.3 & $7.40-7.44(\mathrm{~m})$ & 110.3 & $7.45-7.47(\mathrm{~m})$ \\
\hline $7 ' a$ & 136.9 & & 136.9 & & $136.9^{\mathrm{b}}$ & \\
\hline $1^{\prime \prime}$ & 47.5 & $\begin{array}{l}4.24(\mathrm{td}, \mathrm{J}= \\
7.1,3.8 \mathrm{~Hz})\end{array}$ & 47.5 & $\begin{array}{l}4.28(\mathrm{t}, \mathrm{J} \\
=7.3)\end{array}$ & 44.3 & $\begin{array}{l}4.42(\mathrm{t}, \mathrm{J}= \\
8.3 \mathrm{~Hz})\end{array}$ \\
\hline $2^{\prime \prime}$ & 29.6 & $\begin{array}{l}1.98 \text { (quintet, } \\
7.5 \mathrm{~Hz} \text { ) }\end{array}$ & $\begin{array}{l}26.2 \\
26.3\end{array}$ & $1.96-2.14(\mathrm{~m})$ & 36.2 & $\begin{array}{l}1.86-1.94 \\
(\mathrm{~m}), 2.06- \\
2.14(\mathrm{~m})\end{array}$ \\
\hline $3^{\prime \prime}$ & 23.2 & $\begin{array}{l}1.48^{\mathrm{a}}, \\
\text { overlapped }\end{array}$ & $\begin{array}{l}35.69 \\
35.72\end{array}$ & $\begin{array}{l}1.51^{\mathrm{a}} \text {, } \\
\text { overlapped }\end{array}$ & 70.0 & $3.46-3.52(\mathrm{~m})$ \\
\hline $4^{\prime \prime}$ & 31.9 & $\begin{array}{l}1.63^{\mathrm{a}}, \\
\text { overlapped }\end{array}$ & $\begin{array}{l}67.5 \\
67.6\end{array}$ & $3.85-3.90(\mathrm{~m})$ & 30.8 & $\begin{array}{l}1.52^{\mathrm{a}}, \\
\text { overlapped }\end{array}$ \\
\hline $5^{\prime \prime}$ & 62.3 & $\begin{array}{l}3.67(\mathrm{t}, 6.3 \\
\mathrm{Hz})\end{array}$ & $\begin{array}{l}24.0 \\
24.1\end{array}$ & $\begin{array}{l}1.22(\mathrm{~d}, \mathrm{~J}=6 \\
\mathrm{Hz})\end{array}$ & 9.7 & $\begin{array}{l}0.94(\mathrm{t}, \mathrm{J}= \\
7.5 \mathrm{~Hz})\end{array}$ \\
\hline $1 " \prime$ & 44.0 & $2.38(\mathrm{~s})$ & 44.0 & $2.38(\mathrm{~s})$ & 44.0 & $2.38(\mathrm{~s})$ \\
\hline $2^{\prime \prime \prime}$ & 40.4 & & 40.4 & & $40.4^{b}$ & \\
\hline $3 "$ & 33.2 & & 33.2 & & $33.2^{\mathrm{b}}$ & \\
\hline (E)-2'"-Me & 21.1 & $1.56(\mathrm{~s})$ & 21.1 & $1.56(\mathrm{~s})$ & 21.1 & $\begin{array}{l}1.56^{\mathrm{a}}, \\
\text { overlapped }\end{array}$ \\
\hline (Z)-2"'-Me & 173.6 & & 173.6 & & $173.6^{\mathrm{b}}$ & \\
\hline (E)-3"'-Me & 22.3 & $1.50(\mathrm{~s})$ & $\begin{array}{l}22.26 \\
22.29\end{array}$ & $1.50(\mathrm{~s})$ & 22.3 & $1.49(\mathrm{~s})$ \\
\hline (Z)-3"'-Me & 17.6 & $1.26(\mathrm{~s})$ & $\begin{array}{l}17.61 \\
17.62\end{array}$ & $1.26(\mathrm{~s})$ & 17.6 & $1.26(\mathrm{~s})$ \\
\hline
\end{tabular}

ND not detected

${ }^{a}$ The chemical shifts were taken from HSQC data

${ }^{b}$ The chemical shifts were taken from HMBC data 
Table III. ${ }^{1} \mathrm{H}$ and ${ }^{13} \mathrm{C}$ NMR data for a hydroxy and ketone metabolite (M7) and a carboxy and ketone metabolite (M10) in $\mathrm{CDCl}_{3}$

\begin{tabular}{|c|c|c|c|c|}
\hline \multirow{2}{*}{$\begin{array}{l}\text { Position } \\
\text { No. }\end{array}$} & \multicolumn{2}{|l|}{ M7 } & \multicolumn{2}{|l|}{ M10 } \\
\hline & ${ }^{13} \mathrm{C}$ & ${ }^{1} \mathrm{H}$ & ${ }^{13} \mathrm{C}$ & ${ }^{1} \mathrm{H}$ \\
\hline 1 & 193.4 & & ND & \\
\hline $2^{\prime}$ & 133.9 & $7.78(\mathrm{~s})$ & 136.2 & $7.79(\mathrm{~s})$ \\
\hline $3^{\prime}$ & 119.4 & & $117.4^{\mathrm{b}}$ & \\
\hline $3 ' a$ & 126.3 & & 126.0 & \\
\hline $4^{\prime}$ & 122.8 & $8.38-8.40(\mathrm{~m})$ & 122.7 & $8.32-8.34(\mathrm{~m})$ \\
\hline $5^{\prime}$ & 122.4 & $\begin{array}{l}\text { 7.26-7.30 }(\mathrm{m}), \\
\text { overlapped }\end{array}$ & 123.7 & $\begin{array}{l}\text { 7.35-7.39 }(\mathrm{m}), \\
\text { overlapped }\end{array}$ \\
\hline $6^{\prime}$ & 123.2 & $\begin{array}{l}\text { 7.26-7.30 }(\mathrm{m}), \\
\text { overlapped }\end{array}$ & 124.3 & $\begin{array}{l}7.35-7.39(\mathrm{~m}), \\
\text { overlapped }\end{array}$ \\
\hline $7^{\prime}$ & 109.6 & $7.35-7.37(\mathrm{~m})$ & 110.3 & $7.44-7.47(\mathrm{~m})$ \\
\hline $7^{\prime} \mathrm{a}$ & 136.6 & & $136.9^{\mathrm{b}}$ & \\
\hline $1^{\prime \prime}$ & 45.7 & $4.21(\mathrm{t}, \mathrm{J}=7.3 \mathrm{~Hz})$ & 46.3 & $4.27(\mathrm{t}, \mathrm{J}=7.3 \mathrm{~Hz})$ \\
\hline $2^{\prime \prime}$ & 23.8 & $\begin{array}{l}2.17 \text { (quintet, } \mathrm{J}=6.9 \\
\mathrm{~Hz} \text { ) }\end{array}$ & 23.8 & $\begin{array}{l}2.19 \text { (quintet, } \mathrm{J}=6.9 \\
\mathrm{~Hz} \text { ) }\end{array}$ \\
\hline $3 "$ & 39.6 & $2.43(\mathrm{t}, \mathrm{J}=6.8 \mathrm{~Hz})$ & 39.4 & $\begin{array}{l}2.49(\mathrm{td}, \mathrm{J}=6.4,2.8 \\
\mathrm{Hz})\end{array}$ \\
\hline $4 "$ & 207.5 & & 207.2 & \\
\hline $5^{\prime \prime}$ & 30.0 & $2.11(\mathrm{~s})$, overlapped & 30.1 & $2.15(\mathrm{~s})$ \\
\hline $1^{\prime \prime \prime}$ & 38.8 & $2.11(\mathrm{~s})$, overlapped & 44.0 & $2.37(\mathrm{~s})$ \\
\hline $2^{\prime \prime \prime}$ & 36.3 & & $40.5^{\mathrm{b}}$ & \\
\hline $3^{\prime \prime \prime}$ & 30.4 & & 33.4 & \\
\hline (E)-2'"-Me & 69.5 & $\begin{array}{l}3.77(\mathrm{dd}, \mathrm{J}=38.0, \\
11.0 \mathrm{~Hz})\end{array}$ & 21.1 & $1.57^{\mathrm{c}}$, overlapped \\
\hline (Z)-2"'-Me & 12.4 & $1.44(\mathrm{~s})$ & $173.5^{\mathrm{b}}$ & \\
\hline (E)-3"'-Me & 17.5 or & $1.38(\mathrm{~s})$, overlapped & 22.3 & $1.50(\mathrm{~s})$ \\
\hline (Z)-3"'-Me & $23.5^{\mathrm{a}}$ & $1.38(\mathrm{~s})$, overlapped & 17.6 & $1.26(\mathrm{~s})$ \\
\hline
\end{tabular}

ND not detected

a Based on the data, it could not be determined which chemical shifts correspond to (E)- and (Z)-methyl carbons

${ }^{\mathrm{b}}$ The chemical shifts were taken from HMBC data

${ }^{c}$ The chemical shifts were taken from HSQC data 